\title{
Are the orthostatic fluid shifts to the calves augmented in autonomic failure?
}

\author{
Roland D. Thijs • Adriaan M. Kamper • \\ Arjan D. van Dijk · J. Gert van Dijk
}

Received: 15 March 2009/Accepted: 21 September 2009/Published online: 15 October 2009

(C) The Author(s) 2009. This article is published with open access at Springerlink.com

\begin{abstract}
Background In autonomic failure (AF), blood pressure (BP) falls upon standing which is commonly ascribed to defective vasoconstriction and excessive pooling. Observations on the amount of pooling in AF are contradictory. Methods We evaluated pooling using strain-gauge plethysmography (SGP) during head-up tilt (HUT) with a parachute harness fixed to the tilt table to avoid muscle tension in the lower limbs and thus to maximise pooling. 23 healthy subjects and 12 patients with AF were tilted for $5 \mathrm{~min}$. BP and calf volume changes, as measured by SGP, were measured continuously. Multiple regression analysis was used to examine the effect of AF on orthostatic fluid shifts after adjustment for potential confounders.

Results Patients did not differ from controls with respect to the increase of calf volume after 5 min HUT. The acute $(0-1 \mathrm{~min})$ and the prolonged (1-5 min) phases of calf volume responses to HUT were also similar between patients and controls. No correlation was found between the degree of orthostatic hypotension and the orthostatic calf volume change in AF. In one patient an additional measurement was made before rising from bed in the early
\end{abstract}

R. D. Thijs $(\bowtie)$ · J. G. van Dijk

Department of Neurology and Clinical Neurophysiology, Leiden University Medical Centre, Postal Zone J3-R, PO Box 9600, 2300 RC Leiden, The Netherlands

e-mail: r.d.thijs@lumc.nl; rthijs@sein.nl

\section{A. M. Kamper}

Department of Gerontology and Geriatrics, Leiden University Medical Centre, Leiden,

The Netherlands

A. D. van Dijk

Department of Medical Physics and Engineering,

Leiden University Medical Centre, Leiden,

The Netherlands morning demonstrating a greater albeit small increase of calf volume upon HUT.

Conclusion Orthostatic fluid shifts at the level of the calf in $\mathrm{AF}$ are not augmented during the course of the day despite marked hypotension. However, a small increase of pooling may be expected when the patient first gets out of bed in the morning probably due to the absence of oedema.

Keywords Venous pooling - Orthostatic hypotension . Autonomic failure

\section{Introduction}

Upon standing, 300-1,000 $\mathrm{ml}$ of blood may shift to the lower parts of the body [12, 21, 28, 33]. The time course of orthostatic fluid shifts is characterised by a first fast increase due to filling of veins caused by a rise in hydrostatic pressure and a second slow phase due to fluid filtration through capillary walls $[1,4,21,31]$. The gravitationally induced fluid shifts contribute to the marked differences in pressure in the body upon standing, with a substantial increase in arterial pressure below the heart and a decrease above it [13]. Despite such pressure changes, mean arterial pressure at neck level is maintained in healthy humans mainly through rapidly acting neural reflex mechanisms causing constriction of the capacitance vessels [35]. In autonomic failure (AF), these systems fail and orthostatic hypotension $(\mathrm{OH})$ occurs upon standing. Accordingly, once the gravitational forces are counteracted in AF by standing in water up to the level of the heart or by the inflation of 'antigravity suits', the upright position does not evoke hypotension anymore [11, 16, 30]. $\mathrm{OH}$ is commonly ascribed to defective arteriolar vasoconstriction and excessive venous pooling [28]. As increased orthostatic fluid shifts reduce the venous return to the heart 
and hence may lower blood pressure (BP), a relationship between the degree of $\mathrm{OH}$ and the degree of pooling seems plausible. However, several studies suggested that the orthostatic fluid shifts to the lower limbs in AF are negligible [2, 6, 30, 34]. In contrast, a modest increase of orthostatic pooling to the lower limbs was found in healthy controls after ganglionic blockade [5, 18, 24].

Recently, we described a new method to assess orthostatic fluid shifts with strain-gauge plethysmography (SGP) in the free hanging position [31], and reported a significant gender effect on the amount of pooling to the lower limbs: calf volume upon head-up tilt (HUT) increased more in men than in women. As previous studies on pooling in AF did not control for sex, gender differences may have confounded these results. In addition, it is conceivable that findings were complicated by the time of measurement, i.e. diurnal variation in venous pooling. Leg veins in $\mathrm{AF}$ are exposed to higher than normal intravenous pressure [5], causing the development of oedema during the course of the day [3]. Oedema may function as a "water jacket" around the veins hereby preventing further pooling [19, 32]. Orthostatic fluid shifts are therefore expected to be larger when there is no oedema, i.e. when the patient first gets out of bed and less during the course of the day.

In the present study, we examined whether $\mathrm{OH}$ in $\mathrm{AF}$ is associated with excessive orthostatic fluid shifts at the level of the calf. In addition, we evaluated both the acute and the prolonged phase of orthostatic fluid shifts and in one hospitalised patient we explored the diurnal variation in venous pooling.

\section{Methods}

\section{Subjects}

Thirteen patients with AF were recruited from the outpatient clinic of our tertiary referral centre. Patients were included if they had a history of primary AF and symptomatic orthostatic hypotension, and excluded if they had cardiac disease, varicosities or used antihypertensive medication. One patient with Parkinson's disease was excluded from analysis because of severe dyskinesias causing problems with SGP measurements. Causes of AF in the remaining 12 patients included multiple system atrophy (MSA) $(n=5)$, pure autonomic failure $(n=3)$, Parkinson's disease $(n=1)$, anti-Hu neuropathy $(n=1)$, vincistrine-induced polyneuropathy $(n=1)$ and Sjögren's disease $(n=1)$. One patient used medication for $\mathrm{AF}$ (midodrine $30 \mathrm{mg}$ daily) and slept in the $12^{\circ} \mathrm{HUT}$ position. The other patients were without medication for AF.

Twenty-three healthy volunteers without orthostatic hypotension, recurrent syncope, cardiac disease, varicosities or the use of antihypertensive medication were recruited through an advertisement. The study protocol was approved by the Leiden University Medical Centre ethics review committee. All participants gave written informed consent. All studies were performed in the late morning or the early afternoon. In one hospitalised patient an additional measurement in the early morning was performed to assess the diurnal variation in orthostatic fluid shifts.

\section{Study protocol}

The temperature of the room was maintained at $23 \pm 1{ }^{\circ} \mathrm{C}$. Subjects lay on a motor-driven tilt table (Dewert, $\mathrm{GmbH}$ ). For this experiment the foot board was removed from the table. Tilting time, from $0^{\circ}$ to $60^{\circ}$ head up, was $12 \mathrm{~s}$. While supine, subjects were fitted with a parachute harness fixed to the tilt table to avoid muscle tension in the lower limbs upon tilting. A cushion was placed under the buttocks and a net was attached to the tilt table thus forming a seat. The seat was added to prevent the subject from sliding downwards during HUT and to avoid constriction of the thighs by the leg straps of the harness during HUT. All subjects were tilted with a parachute harness to $60^{\circ}$ head up for $5 \mathrm{~min}$ after at least $5 \mathrm{~min}$ of supine rest.

\section{Measurements}

Both calves were instrumented with mercury-in-silastic strain gauges, placed $10 \mathrm{~cm}$ distally from the tibial tuberosity [31]. The strain gauges were fitted to the measured circumference and connected to a custom-built plethysmograph using the same principles of the Hokanson EC-2 plethysmograph. To avoid direct contact of the strain gauges with the tilt table, the heel was kept away from the table with a small cushion. Beat-to-beat finger BP was measured by finger volume-clamp method (Finometer, Finapres Medical Systems, Arnhem, The Netherlands). Heart rate (HR) was derived from ECG. All signals were routed to a computer (sampling rate $120 \mathrm{~Hz}$ ) for off-line analysis using custom-written software. Volume changes were averaged for both calves. In four patients calf volume measurements of one side were excluded from analysis: two because of previous surgery in that limb and two because of failure to obtain a valid calibration signal.

Data analysis and statistics

Our main outcome measure was the average relative change in calf volume of both legs after 5 min of tilt. Our secondary outcome measures included the calf volume response to the acute phase of tilting ( $0-1$ min HUT), mainly reflecting filling of veins caused by a rise in hydrostatic pressure, and the prolonged phase of tilting 
(1-5 min HUT), mainly reflecting the degree of fluid filtration through capillary walls [28]. The independent sample $t$ test was used to compare the baseline characteristics and the hemodynamic responses to HUT between patients and controls. Significantly different baseline characteristics were considered potential confounders. Multiple linear regression was used to analyse the effect of $\mathrm{AF}$ on orthostatic calf volume changes both before and after adjustment for sex and potential confounders. The association between orthostatic calf volume changes and orthostatic BP changes was assessed using Pearson's correlation. Data analysis was performed with SPSS software, version 12.0. All tests were performed two-sided. Significance threshold was set at $5 \%$.

Table 1 Baseline characteristics and hemodynamic responses to head-up tilt with a parachute harness for patients with autonomic failure $(n=12)$ and healthy controls $(n=23)$

\begin{tabular}{lcc}
\hline & $\begin{array}{c}\text { Patients } \\
(n=12)\end{array}$ & $\begin{array}{c}\text { Controls } \\
(n=23)\end{array}$ \\
\hline Age (years) & $60 \pm 16^{*}$ & $33 \pm 13^{*}$ \\
Length (cm) & $175 \pm 10$ & $177 \pm 10$ \\
Weight $(\mathrm{kg})$ & $78 \pm 11^{*}$ & $70 \pm 9^{*}$ \\
BMI $\left(\mathrm{kg} / \mathrm{m}^{2}\right)$ & $25 \pm 4^{*}$ & $22 \pm 2^{*}$ \\
Baseline calf circumference (cm) & $36 \pm 3$ & $36 \pm 2$ \\
Systolic blood pressure (mmHg) & & \\
$\quad$ Supine & $129 \pm 21$ & $127 \pm 17$ \\
$\quad$ Tilted & $101 \pm 20^{*}$ & $125 \pm 18^{*}$ \\
Diastolic blood pressure (mmHg) & & \\
$\quad$ Supine & $65 \pm 16$ & $68 \pm 13$ \\
$\quad$ Tilted & $56 \pm 11^{*}$ & $75 \pm 13^{*}$ \\
Heart rate (bpm) & & \\
$\quad$ Supine & $76 \pm 8$ & $69 \pm 11$ \\
Tilted & $85 \pm 11$ & $77 \pm 14$ \\
\hline
\end{tabular}

Values are presented as mean $\pm \mathrm{SD}$

* Significance $p<0.05$; independent samples $T$ test

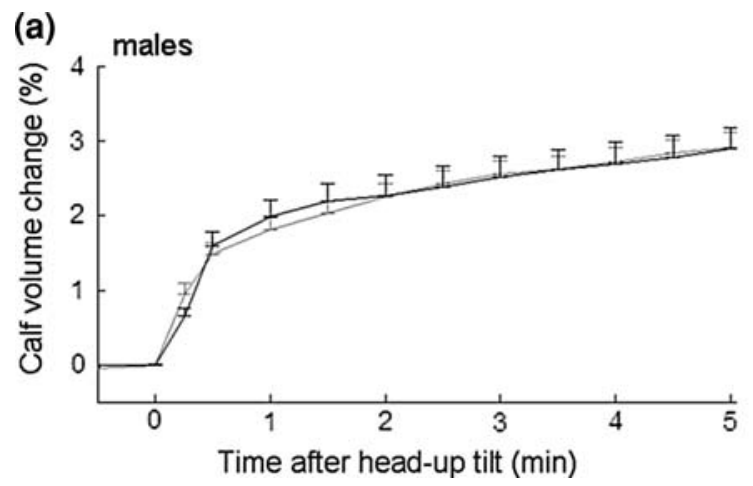

Fig. 1 Time course of calf volume changes in patients (black line) and controls (grey line) as measured by strain-gauge plethysmography during head-up tilt with a parachute harness. Because of a significant

\section{Results}

As shown in Table 1, patients were significantly older, weighed more and had a greater BMI than controls. Patients and controls were similar with respect to length, baseline calf circumference and supine and tilted heart rate. As expected, BP was significantly lower in the HUT position (Table 1). However, patients did not differ from controls with respect to calf volume changes after $5 \mathrm{~min}$ HUT both before and after adjustment for age, sex, weight and BMI (crude 95\% CI for the effect of AF: -0.2 to $1.1 \%$, $p=0.2$; adjusted $95 \%$ CI: -0.8 to $0.7 \%, p=0.9$; Fig. 1 ). Calf volume change in the acute $(0-1 \mathrm{~min})$ and the prolonged (1-5 min) phase of tilting were also similar between patients and controls both before and after adjustment for age, sex, weight and BMI (Table 2). No correlation was found between the degree of orthostatic hypotension and the orthostatic calf volume change in the patients with $\mathrm{AF}$ ( $r=0.14, p=0.7$; Fig. 2$)$.

Of all variables entered in the regression model, only gender significantly affected the calf volume changes induced by 5 min HUT: a greater increase of calf volume upon tilting was seen in men compared to women $(95 \% \mathrm{CI}$ for the difference: $0.4-2.2 \%, p=0.005$ ).

Diurnal variation in orthostatic fluid shifts

A 28-year-old patient with young onset Parkinson's disease was admitted to our hospital because of frequent syncope owing to severe orthostatic hypotension. 24-h BP recording demonstrated marked diurnal BP variations with lowest values in the early morning. Nocturnal weight loss averaged $2 \mathrm{~kg}$. During the course of the day she habitually developed marked ankle oedema. After an afternoon measurement an additional measurement was performed in the early morning. The patient had not been standing upright that morning prior to the experiment except for

(b)

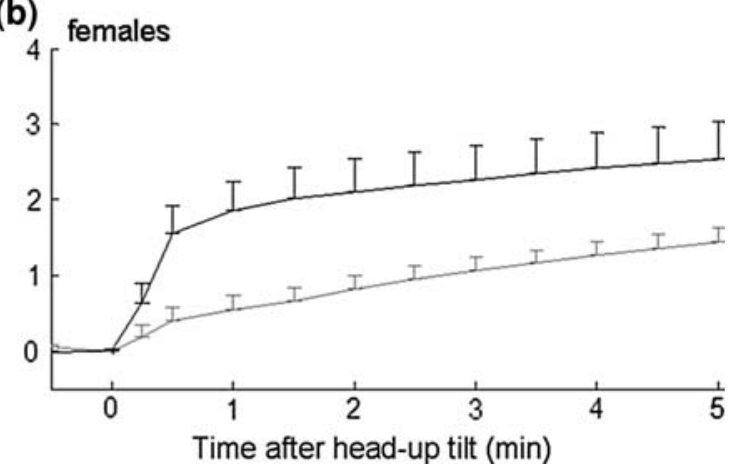

influence of sex on calf volume changes, males (left panel) and females (right panel) are depicted separately. Data are presented as mean \pm SEM 
Table 2 Change of calf volume during head-up tilt (HUT) with a parachute harness in patients with autonomic failure $(n=12)$ and healthy controls $(n=23)$

\begin{tabular}{|c|c|c|c|c|}
\hline & \multicolumn{2}{|c|}{ Autonomic failure } & \multicolumn{2}{|l|}{ Healthy controls } \\
\hline & Men $(n=8)$ & Women $(n=4)$ & Men $(n=14)$ & Women $(n=9)$ \\
\hline Change of calf volume during 5 min HUT (\%) & $2.9 \pm 0.3$ & $2.5 \pm 0.5$ & $2.9 \pm 0.2$ & $1.5 \pm 0.2$ \\
\hline Acute phase $(0-1$ min HUT) & $2.0 \pm 0.2$ & $1.9 \pm 0.4$ & $1.8 \pm 0.2$ & $0.6 \pm 0.2$ \\
\hline Prolonged phase (1-5 min HUT) & $0.9 \pm 0.2$ & $0.7 \pm 0.2$ & $1.2 \pm 0.1$ & $0.9 \pm 0.1$ \\
\hline
\end{tabular}

Data for males and females are shown separately because of gender differences in orthostatic pooling. Values are presented as mean \pm SEM

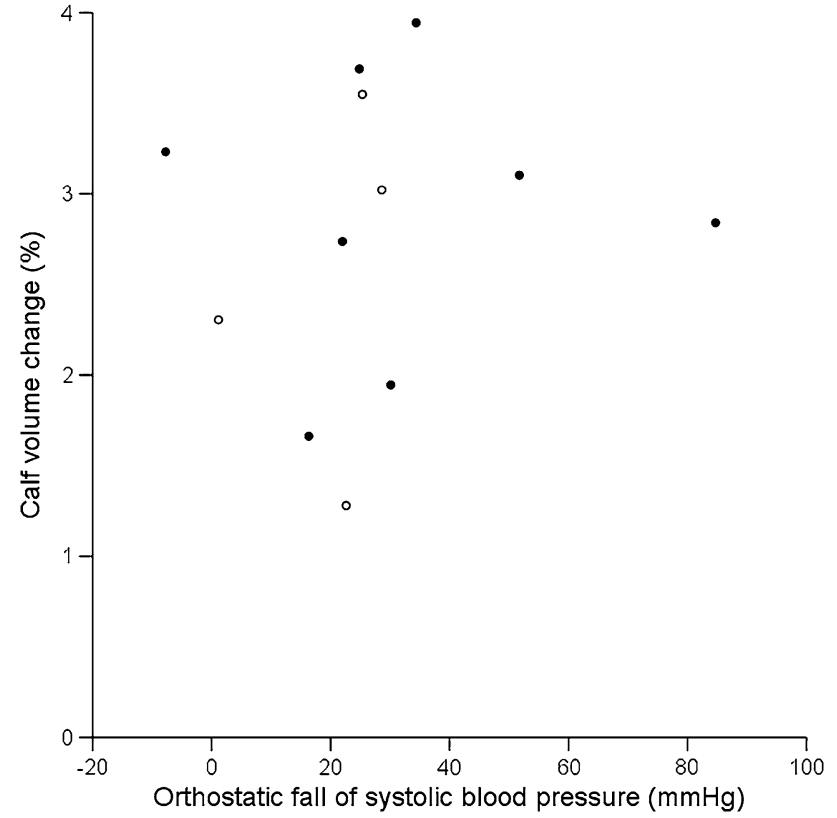

Fig. 2 Relationship between orthostatic fluid shifts at the level of the calf and the orthostatic fall of BP in 12 patients with autonomic failure. No significant correlation was found between the degree of orthostatic hypotension and calf volume changes after 5 min of headup tilt ( $r=0.14, p=0.7)$. Females (open circles) and males (filled circles)

micturition during the night. She had slept with elastic stockings to limit the effect of standing on the amount of pooling. As shown in Fig. 3, BP fall induced by HUT was greatest in the early morning (early morning systolic BP fall $69 \mathrm{mmHg}$ vs. afternoon $23 \mathrm{mmHg}$ ). Calf volume increased more in the early morning $(1.7 \%)$ than in the afternoon (1.3\%). The increase seen in the acute phase of HUT was comparable in both measurements (early morning: $0.7 \%$; afternoon: $1.0 \%$ ), whereas the increase during the prolonged phase of HUT was markedly reduced in the afternoon (early morning 1.0 vs. $0.3 \%$ afternoon).

\section{Discussion}

We found no evidence for excessive pooling at the level of the calf in AF during the course of the day despite marked hypotension. However, the additional measurement in our hospitalised patient suggests that a small increase in orthostatic fluid shifts to the calves may be expected when the patient first gets out of bed in the morning. Since oedema may function as a "water jacket" around the veins $[19,32]$, the observed diurnal variations in pooling are best explained by the development of oedema during the course of the day preventing fluid filtration.

Before discussing the clinical implications of our findings, several limitations to this study deserve mention. First, the degree of AF varied considerably between our patients, as three patients did not meet criteria of $\mathrm{OH}$ at the day of the study (Fig. 2) [9]. One might assume that this explains the lack of venous pooling in AF. However, contrary to expectation, no association was found between the degree of $\mathrm{OH}$ and the amount of orthostatic pooling, a matter further discussed below. Second, age differences between patients and controls may have affected our results. Since venous compliance decreases with age $[15,25]$ and thus may counteract orthostatic pooling, age differences may have caused an underestimation of the amount of pooling in the elder patients compared to the younger controls. The adjustment for age in our regression model cannot completely rule out this bias. However, the amount of $\mathrm{OH}$ in $\mathrm{AF}$ did not correlate with the calf volume changes, thus supporting the notion that $\mathrm{OH}$ should not be ascribed to excessive pooling at the level of the calf. Third, both patients with central and peripheral AF were included. Although both conditions cause $\mathrm{OH}$, preganglionic (central) and postganglionic (peripheral) lesions of the autonomic nervous system cause different types of autonomic dysfunction [7, 36, 37]. The previous finding of a decreased calf compliance, i.e. stiffer veins, in patients with MSA may indicate that pooling is reduced in central AF [17], whereas in peripheral AF the amount of pooling proved to be normal in patients with familial dysautonomia [6] and increased in subjects after ganglionic blockade [5, 18, 24]. Thus, while our study suggests that $\mathrm{OH}$ per se does not cause increased orthostatic fluid shifts to the calves, pooling might differ between central and peripheral AF, but this should be a subject of further study. Fourth, one patient was studied while using $\alpha$ adrenergic medication, 
(a)

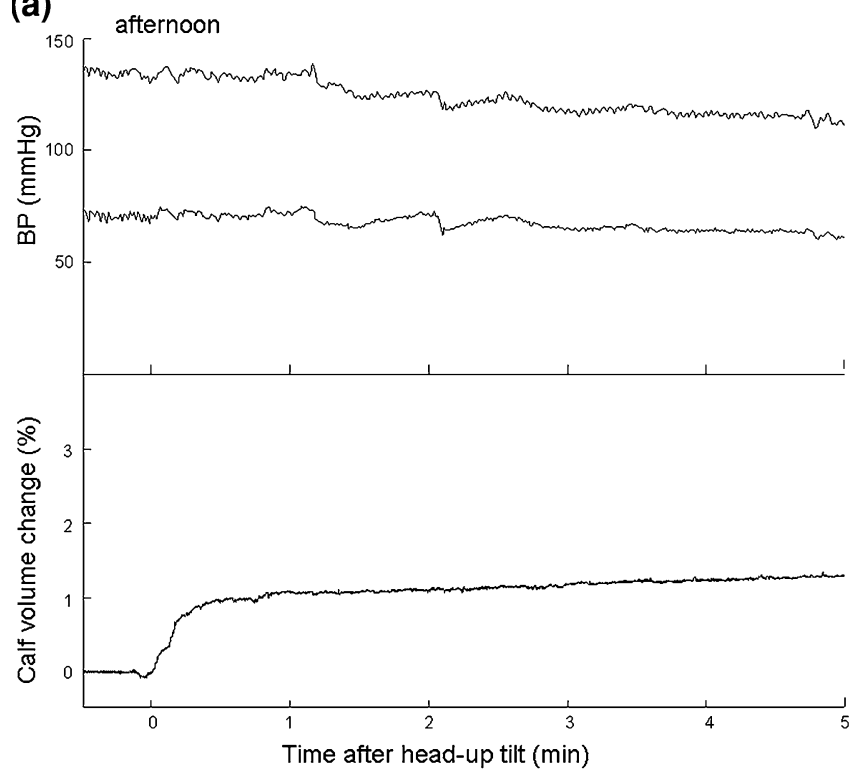

Fig. 3 Orthostatic fluid shifts at the level of the calf during 5 min of head-up tilt with a parachute harness in a 28 -year-old patient with young onset Parkinson's disease. Upon tilting in the afternoon (a) only a small fall of blood pressure (BP) was found (systolic BP fall: $23 \mathrm{mmHg}$ ). This orthostatic fall was accompanied by a $1.3 \%$ increase of calf volume. By contrast, head-up tilt in the early morning (b)

midodrine. Although this decreases calf venous compliance and may thus reduce orthostatic calf volume changes in healthy subjects, the administration of midodrine did not alter venous compliance in patients with MSA [17]. It is therefore not likely that the inclusion of this patient affected our results. Finally, measurements were performed at various times during the day ranging from the late morning to the early afternoon. Given the observed diurnal variation in pooling in our patient with $\mathrm{PD}$, it is conceivable that owing to the development of oedema during the course of the day, a lower amount of pooling to the calves may be found at measurements in the afternoon. Nevertheless, given the relatively small difference between pooling in the early morning and the late afternoon in our patient with $\mathrm{PD}$, the effects of differences in the development of oedema are probably minimal once the patient has risen in the morning.

Calf volume during HUT increased more in men than in women. Gender differences in venous pooling are best explained by a higher venous compliance in men, i.e. more flexible veins, together with an increased hydrostatic pressure due to greater height in men [22, 23, 31]. Surprisingly, as seen in Fig. 1, the difference between both sexes was less in our patients than in healthy subjects. We cannot provide a sufficient explanation for this discrepancy. One could assume that gender differences in healthy subjects are sympathetically mediated, and are thus reduced in case of sympathetic denervation. However, the (b)

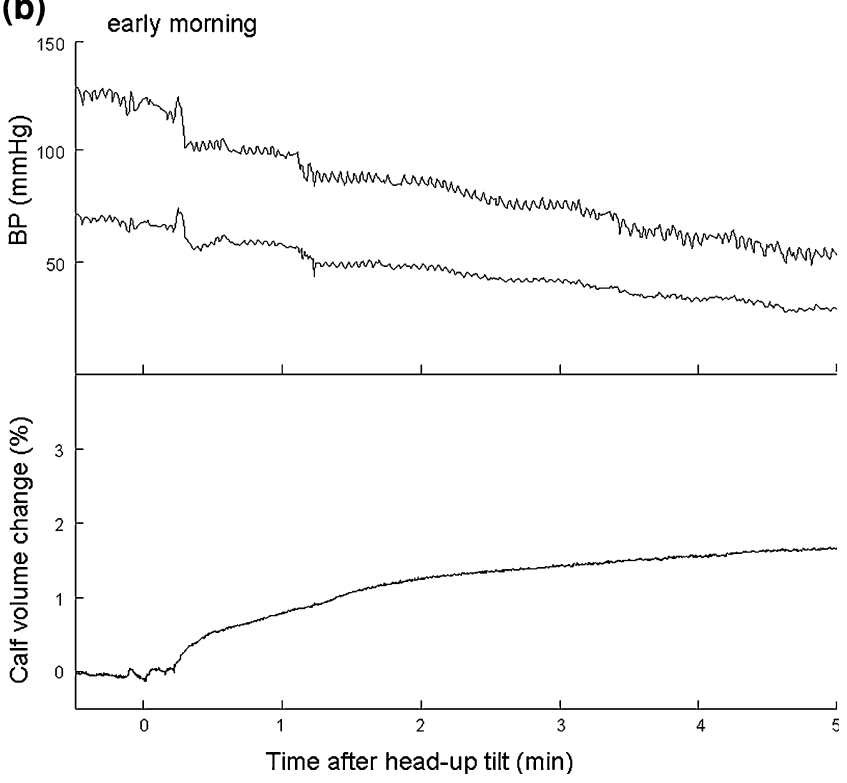

caused severe orthostatic hypotension (systolic BP fall: $69 \mathrm{mmHg}$ ), but only a small increase of calf volume (1.7\%) was seen compared to the afternoon measurements. The observed diurnal variations in pooling are best explained by the development of oedema during the course of the day preventing fluid filtration

gender difference in calf venous compliance was greater during rest compared to sympathetic activation, thus pleading against a sympathetically mediated cause [21]. Alternatively, this discrepancy may be confounded by differences in the amount of $\mathrm{OH}$ within the patients. However, as seen in Fig. 2, the orthostatic BP fall was not greater but lower in female patients.

Although we did not directly assess absolute volume shifts, we can roughly estimate the amount of pooling for the lower leg. Given an average calf volume of $2,077 \mathrm{ml}$ in men [10], a $3 \%$ increase would equal an increase of $62 \mathrm{ml}$ per calf. In confirmation of previous studies, we found no evidence for excessive pooling at the level of the calf in AF. The lack of increased venous pooling at the level of the calf in patients with AF may be explained by the scarce sympathetic innervation of the veins in the lower leg in healthy humans [18]. In contrast, the splanchnic vascular bed is richly innervated and orthostatic pooling in the splanchnic region is probably increased in AF through impaired arterial vasoconstriction [8, 28, 29]. However, the importance of active capacitance responses in the splanchnic bed is difficult to assess in humans and remains debated [14, 27, 28]. Diurnal variations in orthostatic fluid shifts have not been noted previously and should be taken into account in further studies on pooling. The greater amount of pooling when patients rise in the morning may contribute to circadian $\mathrm{BP}$ changes in $\mathrm{AF}$, as patients with $\mathrm{AF}$ have their lowest $\mathrm{BP}$ recordings in the early morning 
$[20,26]$. Nevertheless, it should be stressed that the circadian variation of $\mathrm{BP}$ in $\mathrm{AF}$ cannot be explained by pooling and consequent oedema alone, as confinement to bed did not abolish the daytime BP changes [20].

In conclusion, orthostatic fluid shifts at the level of the calf in AF are not augmented during the course of the day despite marked hypotension. However, a small increase of venous pooling may be expected in the early morning upon first rising from bed, probably due to the absence of oedema. These findings suggest that $\mathrm{OH}$ should not be ascribed to excessive pooling at the level of the calf. Venous pooling in other vascular beds such as the splanchnic region might be of greater importance to $\mathrm{OH}$ in $\mathrm{AF}$.

Acknowledgments We are indebted to R. Fronczek, S. Ober, and R. H. A. M. Reijntjes for their assistance with the preparation of figures.

Open Access This article is distributed under the terms of the Creative Commons Attribution Noncommercial License which permits any noncommercial use, distribution, and reproduction in any medium, provided the original author(s) and source are credited.

\section{References}

1. Asmussen E, Christensen EH, Nielsen M (1940) The regulation of circulation in different postures. Surgery 8:604-616

2. Bannister R, Ardill L, Fentem P (1967) Defective autonomic control of blood vessels in idiopathic orthostatic hypotension. Brain 90:725-746

3. Bradbury S, Eggleston C (1925) Postural hypotension. A report of three cases. Am Heart J 1:73-86

4. Brown CM, Hainsworth R (1999) Assessment of capillary fluid shifts during orthostatic stress in normal subjects and subjects with orthostatic intolerance. Clin Auton Res 9:69-73

5. Brown GE, Wood EH, Lambert EH (1949) Effects of tetra-ethylammonium chloride on the cardiovascular reactions in man to changes in posture and exposure to centrifugal force. J Appl Physiol 2:117-132

6. Brown CM, Stemper B, Welsch G, Brys M, Axelrod FB, Hilz MJ (2003) Orthostatic challenge reveals impaired vascular resistance control, but normal venous pooling and capillary filtration in familial dysautonomia. Clin Sci (Lond) 104:163-169

7. Chandler MP, Mathias CJ (2002) Haemodynamic responses during head-up tilt and tilt reversal in two groups with chronic autonomic failure: pure autonomic failure and multiple system atrophy. J Neurol 249:542-548

8. Chaudhuri KR, Thomaides T, Mathias CJ (1992) Abnormality of superior mesenteric artery blood flow responses in human sympathetic failure. J Physiol 457:477-489

9. Consensus Committee of the American Autonomic Society, the American Academy of Neurology (1996) Consensus statement on the definition of orthostatic hypotension, pure autonomic failure, and multiple system atrophy. Neurology 46:1470

10. Convertino VA, Doerr DF, Flores JF, Hoffler GW, Buchanan P (1988) Leg size and muscle functions associated with leg compliance. J Appl Physiol 64:1017-1021
11. Denq JC, Opfer-Gehrking TL, Giuliani M, Felten J, Convertino VA, Low PA (1997) Efficacy of compression of different capacitance beds in the amelioration of orthostatic hypotension. Clin Auton Res 7:321-326

12. Grubb BP, Karras B (1999) Neurally mediated syncope. In: Mathias CJ, Bannister R (eds) Autonomic failure. A textbook of clinical disorders of the autonomic nervous system. Oxford University Press, Oxford, pp 437-447

13. Hainsworth R (1999) Syncope and fainting: classification and pathophysiological basis. In: Mathias CJ, Bannister R (eds) Autonomic failure. A textbook of clinical disorders of the autonomic nervous system. Oxford University Press, Oxford, pp 428436

14. Hainsworth R, Drinkhill MJ (2006) Counterpoint: active venoconstriction is not important in maintaining or raising end-diastolic volume and stroke volume during exercise and orthostasis. J Appl Physiol 101:1264-1265

15. Hernandez JP, Franke WD (2004) Age- and fitness-related differences in limb venous compliance do not affect tolerance to maximal lower body negative pressure in men and women. J Appl Physiol 97:925-929

16. Jaeger P, Fawer R, Brunner HR, Monti M, Regli F, Schelling JL (1981) Immersion and venous occlusion plethysmography in patients with idiopathic orthostatic hypotension. Clin Exp Hypertens 3:919-927

17. Lipp A, Sandroni P, Ahlskog JE, Maraganore DM, Shults CW, Low PA (2007) Calf venous compliance in multiple system atrophy. Am J Physiol Heart Circ Physiol 293:H260-H265

18. Ludbrook J, Loughlin J (1964) Regulation of volume in postarteriolar vessels of the lower limb. Am Heart J 67:493-507

19. MacLean AR, Allen EV, Magath TB (1944) Orthostatic tachycardia and orthostatic hypotension: defects in the return of venous blood to the heart. Am Heart J 27:145-163

20. Mann S, Altman DG, Raftery EB, Bannister R (1983) Circadian variation of blood pressure in autonomic failure. Circulation 68:477-483

21. Mathias CJ (2002) To stand on one's own legs. Clin Med 2:237245

22. Meendering JR, Torgrimson BN, Houghton BL, Halliwill JR, Minson CT (2005) Effects of menstrual cycle and oral contraceptive use on calf venous compliance. Am J Physiol Heart Circ Physiol 288:H103-H110

23. Monahan KD, Ray CA (2004) Gender affects calf venous compliance at rest and during baroreceptor unloading in humans. Am J Physiol Heart Circ Physiol 286:H895-H901

24. O'Donnel TV (1959) Studies in postural hypotension following ganglion blocking drugs. Clin Sci (Lond) 18:237-248

25. Olsen H, Vernersson E, Länne T (2000) Cardiovascular response to acute hypovolemia in relation to age. Implications for orthostasis and hemorrhage. Am J Physiol Heart Circ Physiol 278:H222-H232

26. Omboni S, Smit AA, van Lieshout JJ, Settels JJ, Langewouters GJ, Wieling W (2001) Mechanisms underlying the impairment in orthostatic tolerance after nocturnal recumbency in patients with autonomic failure. Clin Sci (Lond) 101:609-618

27. Rothe CF (2006) Point: active venoconstriction is/is not important in maintaining or raising end-diastolic volume and stroke volume during exercise and orthostasis. J Appl Physiol 101:1262-1264

28. Smit AA, Halliwill JR, Low PA, Wieling W (1999) Pathophysiological basis of orthostatic hypotension in autonomic failure. J Physiol 519 Pt 1:1-10

29. Smit AA, Wieling W, Fujimura J et al (2004) Use of lower abdominal compression to combat orthostatic hypotension in patients with autonomic dysfunction. Clin Auton Res 14:167-175 
30. Stead EAJ, Ebert RV (1941) Postural hypotension. A disease of the sympathetic nervous system. Arch Intern Med 67:546-562

31. Thijs RD, Bruijnzeels M, Kamper AM, van Dijk AD, van Dijk JG (2007) Assessment of orthostatic fluid shifts with strain gauge plethysmography. Clin Sci (Lond) 113:369-374

32. van Lieshout JJ, Ten Harkel AD, Wieling W (2000) Fludrocortisone and sleeping in the head-up position limit the postural decrease in cardiac output in autonomic failure. Clin Auton Res $10: 35-42$

33. van Lieshout JJ, Wieling W, Karemaker JM, Secher NH (2003) Syncope, cerebral perfusion, and oxygenation. J Appl Physiol 94:833-848

34. Verel D (1951) Postural hypotension: the localization of the lesion. Br Heart J 13:61-67
35. Wieling W, van Lieshout JJ (2008) Maintenance of postural normotension in humans. In: Low PA (ed) Clinical autonomic disorders: evaluation and management. Lippincott Williams \& Wilkins, Philadelphia, pp 57-67

36. Young TM, Mathias CJ (2004) The effects of water ingestion on orthostatic hypotension in two groups of chronic autonomic failure: multiple system atrophy and pure autonomic failure. J Neurol Neurosurg Psychiatry 75:1737-1741

37. Young TM, Asahina M, Nicotra A, Mathias CJ (2006) Skin vasomotor reflex responses in two contrasting groups of autonomic failure: multiple system atrophy and pure autonomic failure. J Neurol 253:846-850 